\title{
A note on the countable chain condition and sigma-finiteness of measures
}

\section{K.P.S. Bhaskara Rao and M. Bhaskara Rao}

The objectives of this paper are the following:

(1) to show that a theorem of Ficker is incorrect;

(2) to show that a stronger version of Ficker's Theorem is valid for a certain class of measures;

(3) characterize all $\sigma$-algebras on which every measure is a countable sum of finite measures.

1. Introduction, notation and definitions

A measure $\mu$ is an extended real valued, nonnegative, countably additive function defined either on a $\sigma$-algebra $A$ of subsets of a set $X$ or on a boolean $\sigma$-algebra $B$ vanishing at the empty set $\emptyset$ or the zero element of $B$. Ficker [1, p. 242] proved the following theorem.

THEOREM (*). Let $\mu$ be a measure on a $\sigma$-algebra $A$ of $X$ and $N$ denote the collection of all sets in A of H-measure zero. Then $A-N$ satisfies countable chain condition (CCC) if and only if $\mu$ can be written as a countable sum of finite measures.

We give an example to show that this Theorem (*) is incorrect.

\section{Example}

Let $B$ be a boolean o-algebra satisfying CCC such that there is no strictly positive, finite measure on $B$. For example, one can take the

Received 16 November 1971. The authors are grateful to Dr Ashok Maitra for useful discussions they had with him on this paper. 
boolean $\sigma$-algebra of all Borel subsets of the real line modulo first category Borel sets. Let $X$ be the Stone space of $B$, A the Baire $\sigma$-algebra on $X$ and $I$ the collection of all first category Baire subsets of $X$. By Loomis' Theorem (see, for example, [2, p. 102]), the quotient boolean $\sigma$-algebra $A / I$ and $B$ are $\sigma$-isomorphic. Since $I$ is a $\sigma$-ideal, the function $\mu$ defined by the formula, $\mu(A)=0$ if $A \in I$, $\mu(A)=\infty$ if $A \in A-I$, is a measure on $A$. Note that $A / I$ satisfies CCC and so $A-I$ satisfies CCC. If Ficker's Theorem (*) were to be true, we can write $\mu$ as a countable sum of finite measures on $A$ which implies that $\mu$ is equivalent to a finite measure $\lambda$ on $A$. Since $I$ is precisely the collection of all $\lambda$-null sets, we have a strictly positive finite measure on $A / I$. But this is a contradiction.

\section{Semi-finite measures}

A measure $\mu$ on a $\sigma$-algebra $A$ of $X$ is said to be semi-finite if $E \in A, \mu(F)=\infty$ implies there exists $E \in A$ such that $E$ is contained in $F$ and $0<\mu(E)<\infty$. For a measure $\mu$ on $A$, there are two definitions of $\mu$-atoms.

(I) A set $A$ in $A$ is said to be a p-atom if

(i) $\mu(A)>0$ and

(ii) $B \in A, B$ contained in $A$ implies $\mu(B)=0$ or $=\mu(A)$.

(II) A set $A$ in $A$ is said to be a $\mu$-atom if

(i) $\mu(A)>0$ and

(ii) $B \in A, B$ contained in $A$ implies $\mu(B)=0$ or $\mu(A-B)=0$.

These definitions are not equivalent. It is easy to construct an example. However, when $\mu$ is semi-finite these two definitions are equivalent. Ficker [1] adopted definition (II) in the course of his proof of the Theorem (*). Under this definition, his Lemma 3 [1, p. 239] is not correct. However, if $\mu$ is semi-finite all his proofs are valid and hence for such a class of measures his Theorem (*) is true.

Here we prove a stronger version of his Theorem (*) directly for semi-finite measures.

THEOREM 1. Let $\mu$ be a semi-finite measure on a o-algebra A of 
$X$. Let $N$ denote the collection of sets of $\mu$-measure zero. Then $A-N$ satisfies CCC if and only if $\mu$ is o-finite.

Proof. If $\mu$ is $\sigma$-finite, it is obvious that $A-N$ satisfies CCC . Conversely, if $\mu(X)<\infty$, there is nothing to prove. If $\mu(X)=\infty$, choose $A_{1}$ in $A$ such that $0<\mu\left(A_{1}\right)<\infty$. Choose $A_{2}$ in $A$ such that $A_{2}$ is contained in $X-A_{1}$ and $0<\mu\left(A_{2}\right)<\infty$. Thus we can find a sequence of disjoint sets $A_{1}, A_{2}, \ldots$ in $A$ such that each $A_{i} \in A-N$ and $\mu\left(A_{i}\right)<\infty$. If $\mu\left(X-\underset{i \geq 1}{U} A_{i}\right)<\infty$, then we have a decomposition of $X$ which implies that $\mu$ is $\sigma$-finite. If $\mu\left(X-\bigcup_{i \geq 1} A_{i}\right)=\infty$, choose $A_{\omega}$ in $A$ such that $A_{\omega}$ is contained in $X-\bigcup_{i \geq 1} A_{i}$ and $0<\mu\left(A_{\omega}\right)<\infty$, where $\omega$ is the first countable ordinal. Continue this process. Since $A-N$ satisfies $\mathrm{CCC}$, there exists a countable ordinal $\alpha$ such that $\mu\left(X-\underset{\beta<\alpha}{U} A_{\beta}\right)<\infty$. This implies that $\mu$ is o-finite.

\section{Some characterizations}

Let $A$ be a $\sigma$-algebra on a set $X$. A set $A$ in $A$ is said to be an atom of $A$ if

(i) $A \neq \varnothing$ and

(ii) $B$ in $A, B$ contained in $A$ implies $B=\emptyset$ or $=A$. A $\sigma$-algebra $A$ on $X$ is said to be atomless if there are no atoms of A .

The following result is known. See Remark 11 of [3, p. 203]. For completeness sake, we give a proof of this result.

PROPOSITION. Let $A$ be a $\sigma$-algebra on a set $X$. A is atomless if and only if every nonempty set in $A$ contains $r_{1}$ disjoint nonempty sets in $A$.

Proof. Let $A$ in $A$ be nonempty. Fix $x \in A$. Find $A_{1}$ in $A$ such that $x \notin A_{1}, A_{1} \neq \varnothing$ and $A_{1}$ is contained in $A$. Choose $A_{2}$ in A such that $x \neq A_{2}, A_{2} \neq \varnothing$ and $A_{2}$ is contained in $A-A_{1}$. 
Continuing this process, we obtain a family $A_{\alpha}: \alpha<\Omega$ of nonempty disjoint sets contained in $A$, where $\Omega$ is the first uncountable ordinal. The converse part is trivial.

THEOREM 2. Let A be a o-algebra on a set $X$. The following statements are all true:

(i) A satisfies $\mathrm{CCC}$ if and only if $\mathrm{A}$ is isomorphic to the power set, that is, the class of all subsets, of some countable (finite or infinite) set;

(ii) there exists a strictly positive finite measure on $A$ if and only if $A$ is isomorphic to the power set of some countable set;

(iii) every measure on A can be written as a countable sum of finite measures if and only if $A$ is isomorphic to the power set of some countable set;

(iv) every measure on $A$ is equivalent to a finite measure if and only if $A$ is isomorphic to the power set of some countable set.

Proof. A proof of (i) can be obtained using the Proposition proved earlier. Since $A$ satisfies $C C C$, the number of atoms of $A$ is countable. From $X$ remove all atoms of $A$. In view of the Proposition the remaining part is empty. The proofs of (ii), (iii) and (iv) are easy.

Professor Ashok Maitra suggested an alternative proof of (i). Since A satisfies CCC, it is complete as a boolean algebra. For $x$ in $X$, the infimum of all sets in A containing $x$ is an atom of A. This implies that $A$ is atomic. Again by CCC, the number of atoms of $A$ is at most countable.

\section{References}

[1] V. Ficker, "On the equivalence of a countable disjoint class of sets of positive measure and a weaker condition than total o-finiteness of measures", Bull. Austral. Math. Soc. 1 (1969), $237-243$. 
[2] Paul R. Halmos, Lectures on Boolean algebras (Van Nostrand, Princeton, New Jersey; Toronto; New York; London; 1963).

[3] B.V. Rao, "On Borel structures", Colloq. Math. 21 (1970), 199-204.

\author{
Research and Training School, \\ Indian Statistical Institute, \\ Calcutta, \\ India.
}

